\title{
Videomicroscopy of methacholine-induced contraction of individ- ual airways in precision-cut lung slices
}

\author{
C. Martin*, S. Uhlig**, V. Ullrich*
}

\begin{abstract}
Videomicroscopy of methacholine-induced contraction of individual airways in precision-cut lung slices. C. Martin, S. Uhlig, V. Ullrich. (C)RS Journals Ltd 1996.

ABSTRACT: Contraction of airways of different size can be studied in viable lung slices by videomicroscopy. However, at present, application of this technique is limited by the heterogeneous responses obtained. We investigated the use of precision-cut lung slices to examine contraction of individual airways.

Lung slices of $250 \pm 20 \mu \mathrm{m}$ were prepared from Wistar rats and cultured in a roller incubator in serum-free minimum essential medium (MEM). Under these conditions, the slices were viable for at least $70 \mathrm{~h}$, as indicated by leakage of lactate dehydrogenase into the supernatant, thymidine incorporation and ciliary beating. The slices were placed in a newly developed incubation chamber and mounted by a nylon thread that was fixed to a platinum wire. The whole chamber was positioned on a microscope stage, and contraction of single airways was followed under a microscope that was coupled to a CCD-camera. Reduction in airway area was taken as an index of bronchoconstriction and was determined by a computer program.

Addition of methacholine resulted in a concentration-dependent (concentration producing half the maximal effect $(\mathbf{E C 5 0})=0.64 \pm 0.08($ mean \pm SD $) \mu M ; n=64)$ contraction of single airways. In the presence of hydrocortisone, the EC50 was about six times greater, i.e. $3.7 \pm 0.9 \mu \mathrm{M}(\mathrm{n}=7)$, and the effect of the steroid was largely abolished by propanolol $(E C 50=1.1 \pm 0.1 \mu M ; n=7)$. Airways with an area smaller than $35,000 \mu^{2}$ were nearly nine times more sensitive to methacholine (EC50 $=0.1 \pm 0.03$ $\mu M ; n=20)$ than larger ones $\left(E_{5}=87 \pm 0.27 \mu M ; n=22\right)$.

We conclude that cultured precision-cut lung slices are a useful model for routine study of contraction of individual airways of various sizes. The measurements were precise and reproducible and showed that smaller airways are more sensitive to methacholine than larger ones.

Eur Respir J., 1996, 9, 2479-2487.
\end{abstract}

*Biological Chemistry, and **Biochemical Pharmacology, University of Konstanz, Konstanz, Germany.

Correspondence: V. Ullrich

Biological Chemistry

University of Konstanz

POB 5560 M611

D-78434 Konstanz

Germany

\section{Keywords: Airway contraction} methacholine

precision-cut lung slices

Received: February 131996

Accepted after revision August 191996
Precision-cut tissue slices have been established as a valuable model system in toxicology. This technique, which was introduced by KRUMDIECK et al. [1], allows the preparation of viable tissue slices of uniform thickness that can be taken into culture [2]. For the lung, an agarose gel instilling technique was developed that enables preparation of tissue cores of this relatively soft tissue [3]. Slices prepared from rat, mouse and human

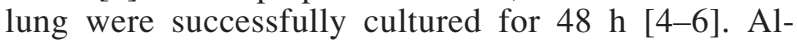
though precision-cut lung slices have been successfully utilized in toxicological studies $[4,5]$, their use in pharmacological or functional studies has not been reported.

DANDURAND et al. [7] have recently demonstrated the principal applicability of lung slices to study airway constriction. With lung slices, constriction of airways can be observed directly under a microscope. Videomicroscopy and digital imaging make it possible not only to visualize but also to quantify bronchoconstriction. For studying bronchoconstriction, this technique, where the histological structure and micromolecular composition of the organ is maintained, seems superior to isolated lung strips or airways. Lung strips in an organ bath may contract in the absence of airway contraction [8-10], and with isolated airways the lack of parenchymal interaction with the airways may be a problem. The isolated perfused lung, though a suitable model for pharmacological studies [e.g. 11], requires an expensive set-up and only one lung can be studied at a time. Another advantage of the lung slice technique is that it makes it possible to study the significance of airway size for bronchoconstriction. The technique of lung slices may be of further interest, since it offers the possibility of investigating human airways obtained by transbronchial biopsy.

In their study, DANDURAND et al. [7] employed lung slices of $500-1,000 \mu \mathrm{m}$ thickness. One problem in their study was the large variation in the constrictor responses of the airways. This may have been due to inherent biological variation (as they argue) or due to variations in slice thickness and/or culture conditions. Precisioncut lung slices make it possible to obtain slices of very reproducible thickness. Therefore, it was of interest to investigate the responses of precision-cut lung slices in 
a set-up that was a further development of that described by DANDURAND et al. [7]. In addition, precision-cut lung slices also have the advantage that they allow very thin slices $(250 \mu \mathrm{m})$ to be investigated. This should make it possible to study smaller airways with higher precision than previously. Finally, Dandurand et al. [7] have always included steroids in their incubation medium, the effect of which is not known under these conditions. For this reason, we also investigated the effect of steroids on airway responsiveness of lung slices. Taken together, the aim of this study was to develop an in vitro model system for studying bronchoconstriction in precision-cut lung slices, and to examine the response to methacholine of airways of various sizes.

\section{Materials and methods}

\section{Animals}

Lungs were taken from 8 week old female Wistar rats (weighing 220 $\pm 20 \mathrm{~g}$ ) obtained from Harlan Winkelman $\mathrm{GmbH}$ (Borchen, Germany) and kept under controlled conditions $\left(22^{\circ} \mathrm{C}, 55 \%\right.$ humidity, $12 \mathrm{~h}$ day/night rhythm) on a standard laboratory chow.

\section{Solutions}

Hank's $\mathrm{Ca}^{2+}$ - and $\mathrm{Mg}^{2+}$-free salt solution was prepared by dissolving $6.9 \mathrm{~g} \cdot \mathrm{L}^{-1} \mathrm{NaCl}, 2.1 \mathrm{~g} \cdot \mathrm{L}^{-1} \mathrm{NaHCO}_{3}$, $0.35 \mathrm{~g} \cdot \mathrm{L}^{-1} \mathrm{KCl}, 0.16 \mathrm{~g} \cdot \mathrm{L}^{-1} \mathrm{KH}_{2} \mathrm{PO}_{4}$ and $1 \mathrm{~g} \cdot \mathrm{L}^{-1}$ glucose in deionized water, with a final $\mathrm{pH}$ of 7.4 after sterile filtration.

Lung slices were cultured in a medium based on minimal essential medium (MEM) (Gibco, Eggenstein, Germany). The MEM powder with Earle's salts was mixed with $20 \mathrm{~mL} \cdot \mathrm{L}^{-1}$ MEM amino acid solution (Gibco), 10 $\mathrm{mL} \cdot \mathrm{L}^{-1}$ of sodium pyruvate (Gibco) and $10 \mathrm{~mL} \cdot \mathrm{L}^{-1}$ vitamin solution (Gibco) and N-2-hydroxyethlypiperazineN'-2-ethanesulphonic acid (HEPES) $5.96 \mathrm{~g} \cdot \mathrm{L}^{-1}$ (Sigma, Deisenhofen, Germany).

For instillation of agarose, a double concentrated HEPES balanced medium was mixed with low melting point agarose solution $(1.5 \% \mathrm{w} / \mathrm{v}$ : Sigma) at a temperature of $40^{\circ} \mathrm{C}$.

\section{Preparation of lung slices}

Lungs were prepared and perfused as described previously [12], with little modifications. After injection of pentobarbital $\left(60 \mathrm{mg} \cdot \mathrm{kg}^{-1}\right)$, the animals were placed under a laminar flow hood and the fur was rinsed with $70 \%$ ethanol. The trachea was cannulated and ventilation was started with a tidal volume of $1 \mathrm{~mL}$ at 80 breaths $\cdot \mathrm{min}^{-1}$. After laparotomy, the diaphragm was removed and heparin (500 IU) was injected into the right ventricle. The animals were exsanguinated by cutting the vena cava. The pulmonary circulation was perfused with Hank's solution via a catheter placed in the pulmonary artery until the lungs were free of blood. The heart and the lungs were removed en bloc and the lungs filled with $10 \mathrm{~mL}$ agarose solution $(0.75 \%$ in MEM, 44 $\left.\mathrm{mL} \cdot \mathrm{kg}^{-1}\right)$ and a bolus of $1 \mathrm{~mL}$ air [7]. After cooling of the agarose to $4^{\circ} \mathrm{C}$, tissue cores were prepared by advancing a rotating sharpened metal tube (diameter $8 \mathrm{~mm}$ ). From these cores, tissue slices $(250 \pm 20$ (mean \pm SD) $\mu \mathrm{m})$ were prepared using a Krumdieck tissue slicer (Alabama Research and Development, Munford, AL, USA). The thickness of the slices was determined by means of an optical micrometer.

\section{Culture of tissue slices}

Lung slices were floated on Teflon mesh inserts (two slices per insert) and cultured in glass vials containing $1 \mathrm{~mL}$ of MEM. The vials were placed on a Roller system housed in a humidified incubator. They were incubated at $37^{\circ} \mathrm{C}$ in a humid atmosphere and rotated at 10 rpm. The medium was changed every hour during the first $4 \mathrm{~h}$ and, thereafter, every $24 \mathrm{~h}$. Alternatively, in some experiments lung slices were incubated in 24-well plates.

\section{Incubation on the stage of the microscope}

An incubation chamber (fig. 1) was developed to allow incubation and observation of slices by an inverted microscope. The chamber was made of polycarbonate and connected through inlet $(\mathrm{A})$ and outlet $(\mathrm{F})$ to a waterbath. Two incubation cells (C) were positioned in the centre of the chamber. The bottom was sealed by glass $(\mathrm{G})$ and the cover by acrylic glass $(\mathrm{H})$. The slices were fixed in the incubation cells by positioning them

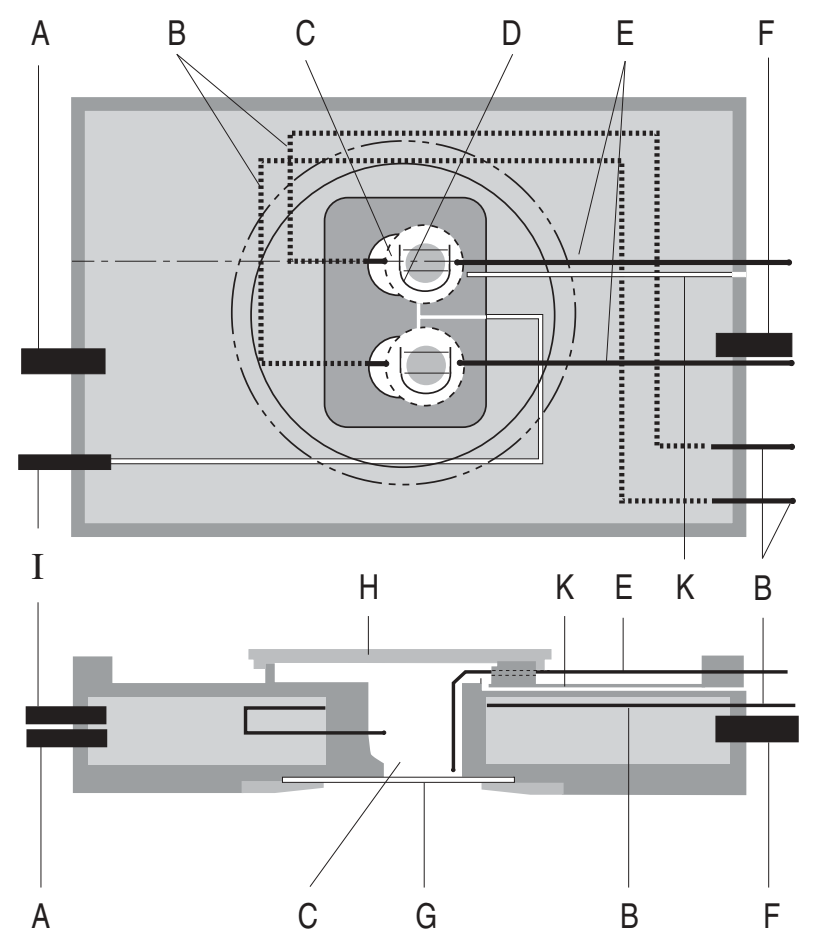

Fig. 1. - Incubation chamber in a) plan view and b) cross section to be fixed on the microscope stage. The chamber was connected to a waterbath through inlet $\mathrm{A}$ and outlet $\mathrm{F}$; $\mathrm{B}$ : filling pipe; $\mathrm{C}$ : incubation cell; D: platinum wire with nylon threads; E: vacuum pipe; G: glass bottom; H: cover; I: gas inlet; K: gas outlet. 
under nylon strings, that were fixed to a bent platinum wire (D). The incubation cells (C) could be filled with buffer, medium or drug solutions through the filling pipe (B). Buffer solution could be removed from the incubation cell by negative pressure applied to a vacuum pipe (E). Inlet (I) and outlet (K) enabled aeration of the incubation cell.

\section{Image acquisition}

The incubation chamber was placed on the stage of an inverted microscope (Zeiss Axiovert 35, Oberkochen, Germany) and warmed to $37^{\circ} \mathrm{C}$. The slices were screened for airways and transferred to the incubation chamber. Lung slices were selected for study, using criteria similar to those used by DANDURAND et al. [7], only if: 1) they contained at least one airway; 2) the entire epithelial-luminal junction of the airways was in focus; 3 ) the airways were free of agarose and debris; and 4) beating of cilia was observed. The airways were focused, imaged with a video camera (Kappa CF8RCC; Kappa, Gleichen, Germany), and digitized using a frame grabber board (CFG-KIT-C2 AT; Imaging Technology Inc., Bendford, MA, USA). The images were saved on a hard disk. An image of $2.8 \mathrm{~mm}^{2}$ was represented by $512 \times 768$ pixels.

After preincubation for $10 \mathrm{~min}$ with $1 \mathrm{~mL}$ of MEM, the first image was acquired. The airway area obtained from this first image served as the reference area (100\%). The liquid was removed, and methacholine (MCh) diluted in $1 \mathrm{~mL}$ of medium was transferred into the incubation cell. The airway was imaged every minute during the 10 min incubation time, when the bronchoconstriction was always maximal.

The MCh concentration was varied from $10^{-10}$ to $10^{-2}$ M. In the first study, a new slice was used for each concentration. In the second study, all concentrations were measured on one slice, i.e. cumulative concentrationresponse curves were constructed.

\section{Image analysis}

The images were analysed by an image analysis program (Optimas 5.2; Optimas Corporation Bothell, Washington, USA). The lumen area was taken as the area enclosed by the epithelial luminal border and was quantified by the Optimas software package after setting the appropriate threshold. Control airway area was defined as $100 \%$.

\section{Viability tests}

Viability was assessed by measuring leakage of lactate dehydrogenase (LDH) from tissue slices into the supernatant. At appropriate time-points, slices were removed from the roller incubator and transferred to $0.2 \%$ Triton $\mathrm{X}-100$ solution. All samples were stored over night at $4{ }^{\circ} \mathrm{C}$, minced and sonicated $3 \mathrm{~h}$ prior to the LDH measurement. LDH activity was measured both in the incubation medium and the slice, and expressed as the percentage of $\mathrm{LDH}$ in the supernatant $(\mathrm{S})$, according to the following equation: \% LDH in $\mathrm{S}=100(\mathrm{~S}) /(\mathrm{S}+$ slice). LDH activity was measured as described by HORDER and REJ [13] using an Eppendorf (Hamburg, Germany) ACP 5040 enzyme analyser.

Alternatively, viability was assessed by measuring thymidine incorporation. Lung slices were incubated with $1 \mu \mathrm{Ci}\left[{ }^{3} \mathrm{H}\right]$ thymidine $\left(85 \mathrm{Ci} \cdot \mathrm{mmol}^{-1}\right.$; Amersham, Braunschweig, Germany) for $4 \mathrm{~h}$ in the incubation roller. After incorporation of the label, the slices were washed twice with phosphate-buffered saline (PBS), transferred to $0.5 \mathrm{~mL} 0.2 \%$ Triton $\mathrm{X}-100$ solution and minced. Ten microlitre aliquots of the homogenates were analysed for protein using a Bradford protein assay kit (Biorad, München, Germany). The remainder of the homogenates were assayed in a gamma counter.

Measurement of 3-[4.5-dimethylthiazol-2-yl]-2,5-diphenyltetrazolium bromide (MTT), as an index of mitochondrial activity was performed according to MOSMAN [14]. After incubation in dynamic organ cuture the slices were transferred into 24 -well plates and were incubated with $0.7 \mathrm{mg} \cdot \mathrm{mL}^{-1}$ MTT for $15 \mathrm{~min}$. After removal of medium, the slices were lysed for $20 \mathrm{~min}$ in $200 \mu \mathrm{L}$ i-propanol/formic acid (95/5). One hundred microlitres of the solution containing lysed lung cells was transferred into 96-well microtitre plates. The reduced MTT was measured spectrophotometrically in an enzymelinked immunosorbent assay (ELISA) reader at 560/690 $\mathrm{nm}$.

\section{Statistical analysis}

Data are expressed as mean \pm SD. Sigmoidal concentration-response curves were fitted by using the fourparameter logistic equation program, Allfit [15]. This program allows simultaneous analysis and comparison of families of sigmoidal curves. The concentration causing $50 \%$ of maximal response (EC50) values and Hill slopes for concentration-response curves were obtained from this program. Identity of EC50 values and slopes was tested for by comparing the sum of squares from the constrained and the unconstrained model (Ftest; $\mathrm{p}<0.05)$. Therefore, if different EC50 values are given within one group, they were significantly different from each other $(\mathrm{p}<0.05)$.

\section{Results}

\section{Viability}

Slices of reproducible thickness of $250 \pm 20 \mu \mathrm{m}(\mathrm{n}=40)$ were obtained. They were incubated in MEM buffer free of steroids and serum, and their viability was studied. Leakage of LDH into the supernatant was high during preincubation and decreased rapidly with time. LDH leakage remained below $5 \%$ per $24 \mathrm{~h}$ for up to 3 days. Assessment of LDH leakage was used to compare the viability of lung slices incubated in a roller incubator to those incubated in typical cell culture wells. LDH leakage of the slices incubated in wells was approximately $15 \%$ per $24 \mathrm{~h}$ (fig. 2). These results suggest that culturing of slices in a roller incubator is preferable to incubation in wells. 


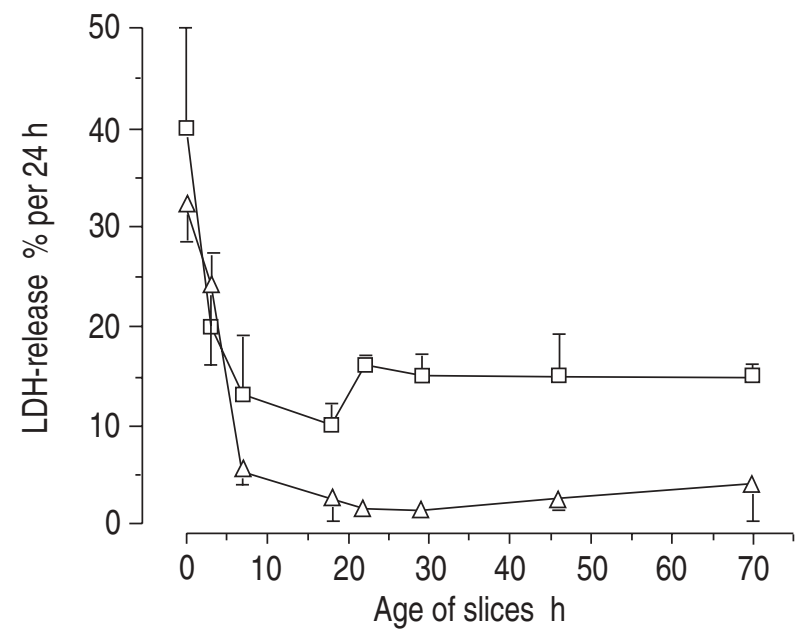

Fig. 2. - Time-dependent leakage of lactate dehydrogenase (LDH) from rat lung slices under different culturing conditions. Slices were incubated in 24-well plates $(\square)$ and in a roller incubation system $(\Delta)$. Data are mean \pm SD from 15 experiments. Comparing the two conditions all values shown were significantly different at the level $\mathrm{p}<0.05$ except time point two, at 3 hours (unpaired t-test).

\section{Viability}

Thymidine incorporation was tested as a proliferation assay. Figure 3 shows thymidine incorporation for 3 days. After $24 \mathrm{~h}$ of incubation, there was a marked increase in thymidine incorporation, which remained at this level for up to $72 \mathrm{~h}$. To test for mitochondrial function, the ability of lung slices to reduce MTT was investigated. Reduction of MTT depends on the activity of the mitochondrial enzyme, succinate dehydrogenase. Figure 4 shows that an inhibitor of succinate dehydrogenase, i.e. 3-nitropropionate, concentrationdependently inhibited MTT reduction in lung slices. The calculated median inhibitory concentration (IC50) of $6.3 \times 10^{-4} \mathrm{M}$ was similar to that described previously, i.e. $2 \times 10^{-4} \mathrm{M}$ [16]. The ability of lung slices to reduce MTT was maintained for at least 3 days (data not shown). These data, i.e. LDH leakage, thymidine incorporation and MTT reduction suggest that slices were viable for up to $72 \mathrm{~h}$.

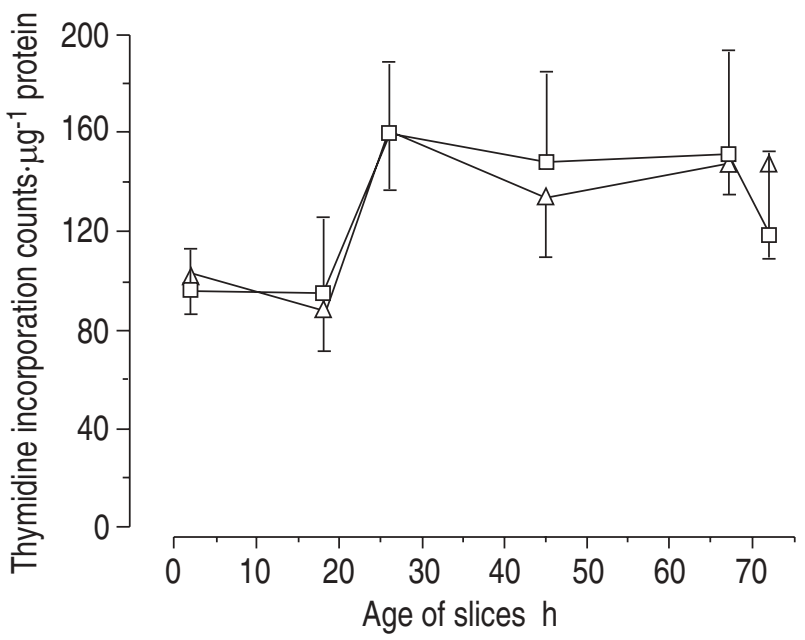

Fig. 3. - Time-dependent incorporation of $\left[{ }^{3} \mathrm{H}\right]$-thymidine in the rat lung slices. Slices were incubated in 24-well plates ( $\square$ ) and in a roller incubation system $(\Delta)$. Data are mean \pm SD from nine experiments.

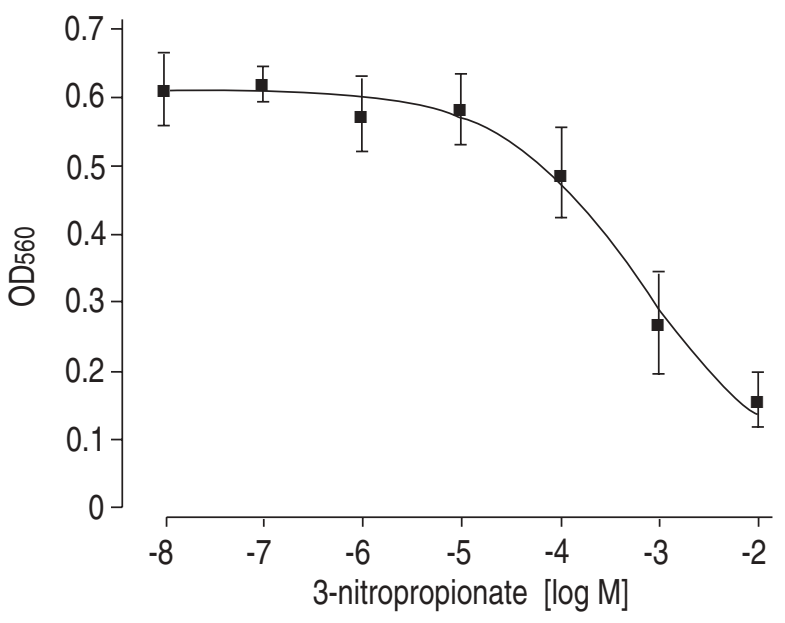

Fig. 4. - Concentration-dependent inhibition of MTT reduction in lung slices by 3-nitropropionate, measured at an optical density of $560 \mathrm{~nm}$ (OD560). Lung slices were preincubated with 3-nitropropionate acid for $14 \mathrm{~h}$, before MTT reduction was measured as indicated in Materials and Methods. The IC50 for inhibition of MTT reduction by 3 -nitropropionate was $6.3 \times 10^{-4} \mathrm{M}$. Data are mean \pm SD from six experiments. MTT: 3-(4,5-dimethylthiazol-2-yl)-2,5-diphenyltetrazolium bromide; IC50: median inhibitory concentration.

\section{Contractility}

The effect of MCh on one individual airway and vessel is demonstrated in figure 5. Figure 5a shows a lung slice including an airway and a vessel. After $10 \mathrm{~min}$ preincubation under baseline conditions, control airway area and vessel area were measured and defined as $100 \%$. The slice was imaged again $10 \mathrm{~min}$ after treatment with $\mathrm{MCh}$ concentrations from $10^{-10}$ to $10^{-4} \mathrm{M}$. The corresponding concentration-response curves of airway and vessel area are shown in figure $5 \mathrm{~b}$. A decrease in airway area was observed between MCh concentrations of $10^{-7}$ to $10^{-5} \mathrm{M}$. The vessel area did not change significantly with the concentrations used.

To compare the effect of cumulative treatment compared to single treatment on MCh-induced bronchoconstriction table 1: in Study 1 a new slice was used for each concentration; and in Study 2 the concentration of methacholine was raised in a stepwise manner, while the same slice was used. The EC50 values and the Hill slopes in Studies 1 and 2 were not significantly different $(\mathrm{p}>0.05)$. From these data, we conclude that cumulative concentration-response curves are practicable.

We next investigated $\mathrm{MCh}$-induced bronchoconstriction depending on the age of the slices, i.e. incubation time in the roller incubator table 2 . Three periods of incubation time were selected: $5-8 \mathrm{~h} ; 15-18 \mathrm{~h}$; and $24-28$ $\mathrm{h}$. The EC50 values for the three incubation periods were not significantly different $(\mathrm{p}>0.05)$. These observations provide evidence that lung slices remain functionally intact for up to $28 \mathrm{~h}$.

\section{Relaxation}

The responses of isolated airways may depend on initial intrinsic tone [17]. Therefore, we investigated the response of lung slices to common bronchodilators, such as phosphodiesterase (PDE) inhibitors, $\beta_{2}$-agonists and 
a)
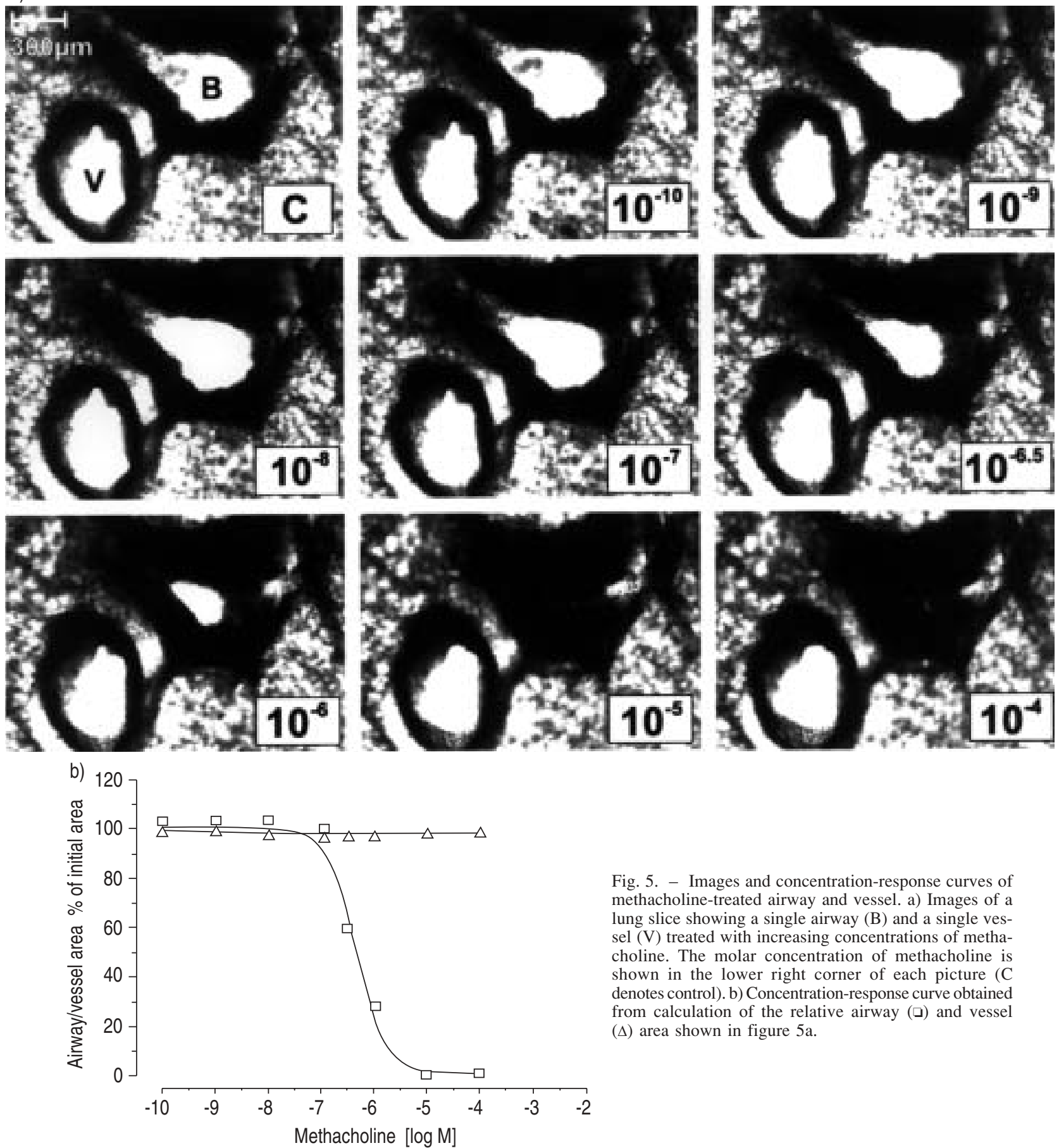

Fig. 5. - Images and concentration-response curves of methacholine-treated airway and vessel. a) Images of a lung slice showing a single airway (B) and a single vessel (V) treated with increasing concentrations of methacholine. The molar concentration of methacholine is shown in the lower right corner of each picture (C denotes control). b) Concentration-response curve obtained from calculation of the relative airway ( $\square$ ) and vessel $(\Delta)$ area shown in figure $5 \mathrm{a}$

forskolin. Incubating lung slices with the $\beta_{2}$-agonists, salbutamol and the PDE-inhibitor 3-isobutyl-1-methylxanthine (IBMX), caused no change in airway area of

Table 1. - Comparison of single and multiple treatment of lung slices with MCh

\begin{tabular}{lccc}
\hline & $\begin{array}{c}\text { Age of slices } \\
\mathrm{h}\end{array}$ & $\begin{array}{c}\text { EC50 } \\
\mu \mathrm{M}\end{array}$ & Hill-slope \\
\hline Study 1 & $5-8$ & $0.75 \pm 0.05$ & 1.29 \\
Study 2 & $5-8$ & $0.62 \pm 0.04$ & 1.28 \\
\hline
\end{tabular}

MCh: methacholine; EC50: concentration producing half the maximal effect. untreated lung slices (fig. 6). These concentrations of salbutamol/IBMX were, however, effective in our model as shown by their ability to reverse MCh-induced airway

Table 2. - Influence of culturing time on MCh-induced airway contraction

\begin{tabular}{ccc}
\hline $\begin{array}{c}\text { Age of slices } \\
\mathrm{h}\end{array}$ & $\begin{array}{c}\text { EC50 } \\
\mu \mathrm{M}\end{array}$ & Hill-slope \\
\hline $5-8$ & $0.62 \pm 0.04$ & \\
$15-18$ & $0.64 \pm 0.07$ & 1.29 \\
$24-28$ & $0.64 \pm 0.08$ & 1.29 \\
\hline
\end{tabular}

MCh: methacholine; EC50: concentration producing half the maximal effect. 


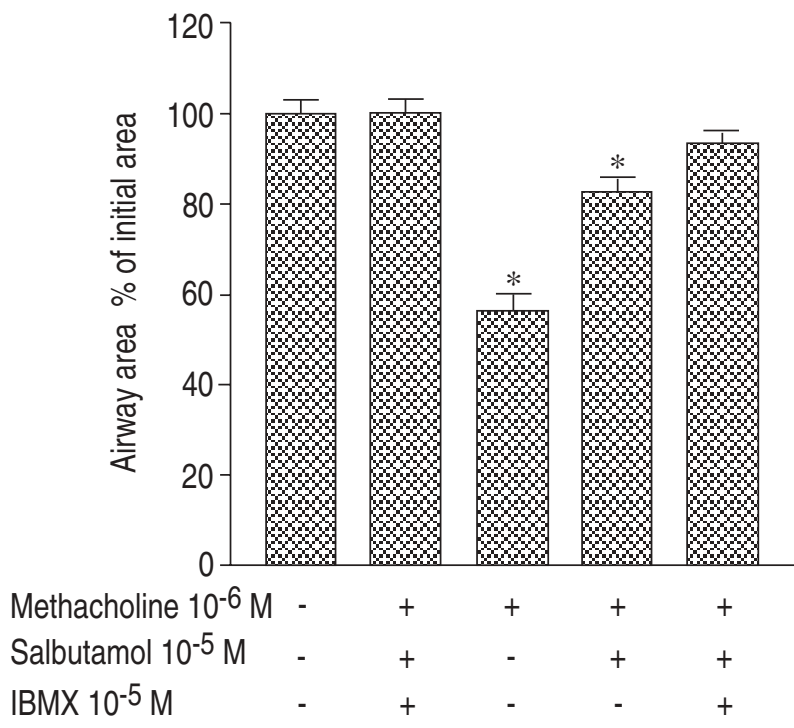

Fig. 6. - Relaxation of airways by salbutamol and IBMX. Single airways were incubated under the conditions indicated in the figure. Mean airway size was $152,000 \pm 46,636 \mu \mathrm{m}^{2} ; \mathrm{n}=3$. *: $\mathrm{p}<0.05$, compared to Control (first bar) by Dunnett's test for multiple comparisons. IBMX: 3-isobutyl-1-methylxanthine.

constriction (fig. 6). These data suggest that no intrinsic tone was present in the airways of the lung slices.

\section{Steroids}

In contrast to the present study, others [4, 7] have routinely included glucocorticoids in their incubations. We therefore investigated whether glucocorticoids influence MCh-induced bronchoconstriction in lung slices. Slices were incubated for $24 \mathrm{~h}$ in the presence or absence of $0.1 \mu \mathrm{g} \cdot \mathrm{mL}^{-1}$ hydrocortisone $(\mathrm{HC})$. The measurement

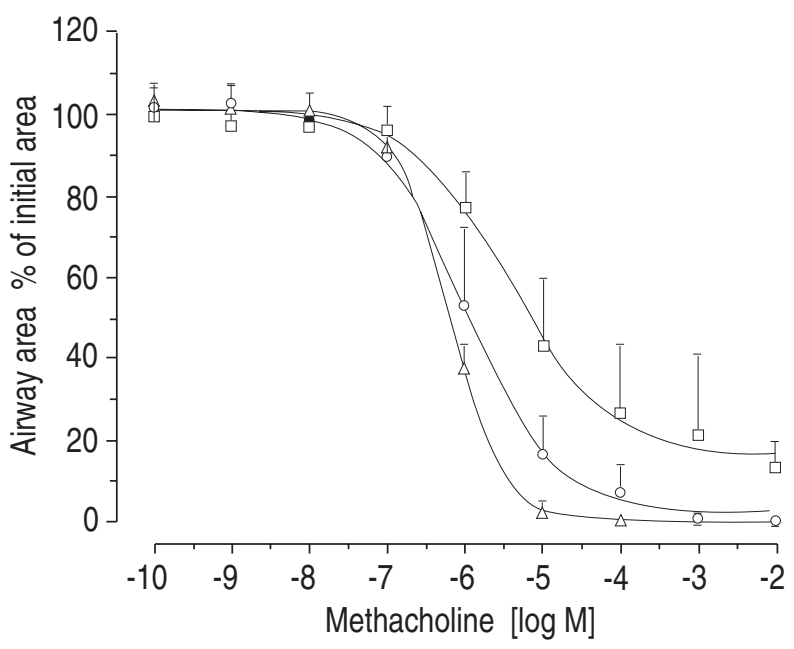

Fig. 7. - Influence of hydrocortisone on methacholine-induced bronchoconstriction in rat lung slices. The slices were incubated in the presence ( $\square ; \mathrm{n}=42$ for whole curve); or absence $(\Delta ; \mathrm{n}=52$ for whole curve) of $0.1 \mu \mathrm{g} \cdot \mathrm{mL}^{-1}$ hydrocortisone for $24 \mathrm{~h}$ before treatment with methacholine (MCh). MCh induced bronchoconstriction in slices incubated with $\mathrm{HC}$ in the presence of propanolol $\left(10^{-6} \mathrm{M}\right)(\mathrm{O} ; \mathrm{n}=48$ for whole curve). As calculated by the program Allfit, all three curves were significantly different from each other. Data are mean \pm SD. of bronchoconstriction itself was performed in MEM in the absence of HC. Even at the highest MCh concentration used, HC-treated airways contracted only incompletely ( $15 \%$ of airway area). The concentration-response curve was shifted to the right. The EC50 value for MChinduced contraction for airways from slices incubated with and without $\mathrm{HC}$ was $3.7 \pm 0.86$ and $0.64 \pm 0.08 \mu \mathrm{M}$, respectively (fig. 7). Slices that were incubated for 24 $\mathrm{h}$ in MEM with $\mathrm{HC}$ and preincubated with $1 \mu \mathrm{M}$ propanolol for $10 \mathrm{~min}$ prior to addition of MCh showed a reinforced contraction in response to $\mathrm{MCh}(\mathrm{EC} 50=1.1 \pm$ $0.11 \mu \mathrm{M}$ ) (fig. 7). Since airway responsiveness may depend on airway size (see below), it was important to ascertain that the area of all airways in these studies was comparable: control 151,279 $\pm 28,296 \mathrm{um}^{2}$, HC 142,656 \pm

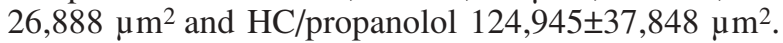
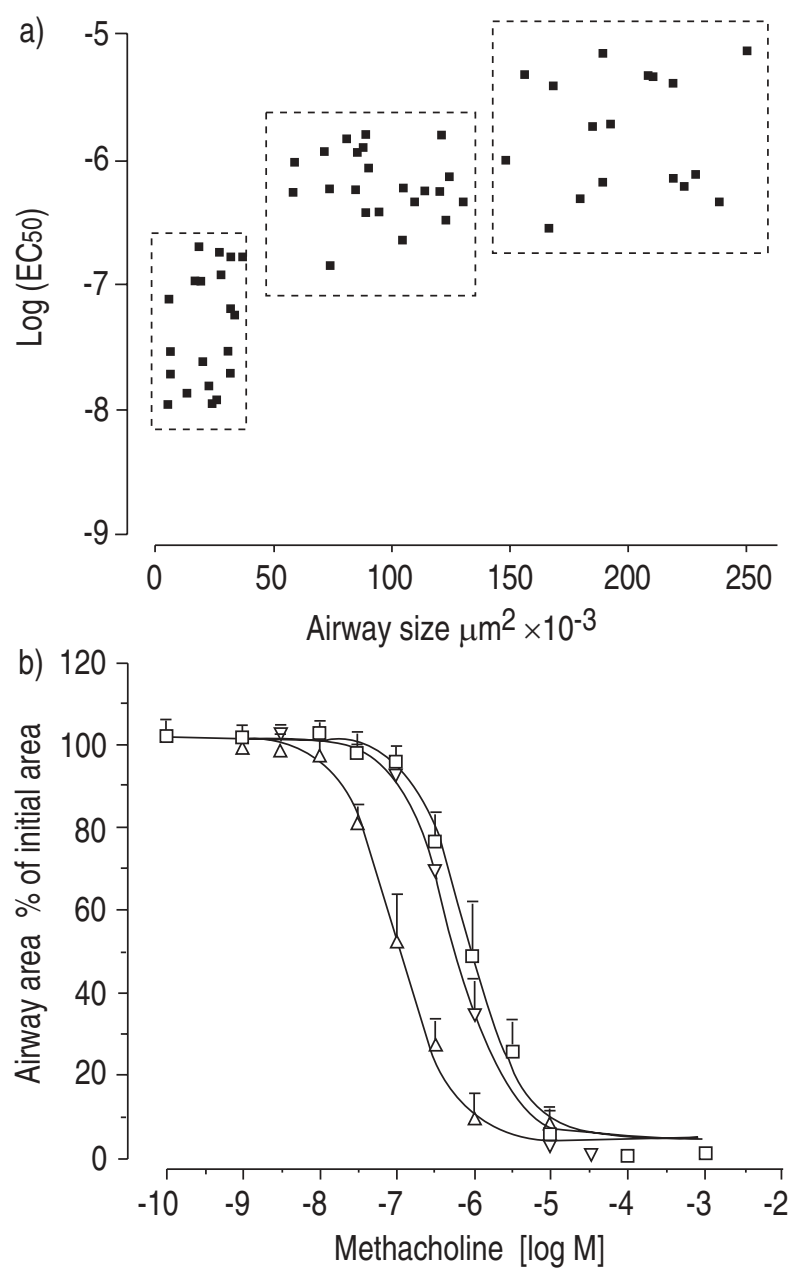

Fig. 8. - Methacholine-induced bronchoconstriction in rat lung slices depending on airway size. a) Correlation diagram of airway size plotted against log EC50 from all experiments. Each dot represents the EC50 for methacholine-induced airway contraction at the given airway size. The dashed boxes indicate the three different groups that were formed for further analysis, i.e. small $\left(<35,000 \mu \mathrm{m}^{2}\right)$, medium $\left(35,000-130,000 \mathrm{~m} \mathrm{~m}^{2}\right)$ and large airways $\left(>130,000 \mu \mathrm{m}^{2}\right)$. b) Concentration-response curves for methacholine-induced bronchoconstriction in small $(\Delta)$, medium-sized $(\nabla)$ and large $(\square)$ airways. The corresponding EC50 values were $0.1,0.56$ and $0.87 \mu \mathrm{M}$, respectively. Each curve was calculated from 76-89 data points obtained from 20-22 different lung slices. EC50: concentration producing half the maximal effect. Data are mean \pm SD. 


\section{Airway size}

In order to examine the dependence of bronchoconstriction on airway size, airways ranging 1,000-260,000 $\mu^{2}$ were studied in 64 slices. MCh concentrations were varied from $10^{-9}$ to $10^{-3} \mathrm{M}$, and IC50 values were calculated for each slice. Figure 8a shows a correlation diagram of airway size plotted against EC50. Since no linear relationship appears to exist between airway size and the EC50 values, the data were analysed by nonparametric regression and a Spearman rank correlation coefficient of $0.79(\mathrm{p}<0.0001)$ was calculated.

To further analyse these data, airways were divided into three groups: small, medium-sized and large, as indicated in figure 8a. Concentration-response curves for the MCh-induced bronchoconstriction of these three groups are shown in figure $8 \mathrm{~b}$. Statistical analysis (Allfit program) showed that all three dose-response curves had common maximum and minimum values, as well as a common slope factor. Similar slopes of the curves suggest similar mechanisms; similar minima show that the maximal contraction was equal in large, medium and small airways. The following EC50 values were determined: small airways $0.10 \pm 0.03 \mu \mathrm{M}$; medium-sized airways $0.56 \pm 0.30 \mu \mathrm{M}$; large airways $0.87 \pm 0.27 \mu \mathrm{M}$. For medium-sized and large airways, the EC50 values were not significantly different $(\mathrm{p}>0.1)$. In contrast, the EC50 value obtained for small airways was significantly different from that of both the medium-sized and the large airways $(\mathrm{p}<0.05)$. At a MCh concentration of $0.1 \mu \mathrm{M}$ small airway area was reduced to $50 \%$, whereas medium-sized and large airways showed no significant airway contraction at this concentration. From these data, we conclude that $\mathrm{MCh}$-induced bronchoconstriction is dependent on airway size. Small airways contract at lower MCh concentrations than medium-sized or large airways.

\section{Discussion}

This study describes a method for the measurement of bronchoconstriction in precision-cut lung slices. The anatomical structure was preserved and the slices could be used for pharmacological studies for at least $28 \mathrm{~h}$. The set-up developed in this study allows direct observation of airway contraction in small and large airways. The method allows rapid screening of drugs for bronchoconstriction and determination of EC50, values. Each lung provides about 20 viable slices including airways, which permits screening of up to six different drugs at a time. In principle, measurement of vasoconstriction is also possible, although it is difficult to discriminate between the pulmonary artery, the pulmonary vein and the bronchial artery.

A new incubation chamber for use under a microscope was developed. DANDURAND et al. [7] described a method in which lung slices were incubated in well plates and, subsequently, the plates were transferred to a microscope and bronchoconstriction was measured. The shortcomings of this method were identification of airways, floating of slices and, therefore, limited magnification under the microscope. To overcome these problems, slices were mounted in the incubation chamber by nylon threads fixed to a platinum wire. The use of thin nylon threads was particularly important to avoid interference with the observation area of the microscope. The weight of the platinum wire was chosen such that both moving and injury of lung slices was avoided. This system allows microscopy under $\times 400$ magnification, which is essential for the observation of small airways (diameter $<50 \mu \mathrm{m}$ ). The incubation chamber enables aeration and warming of the medium in the incubation cell on the microscope stage. This makes it possible to incubate the slices in bicarbonate buffer and to observe slices for longer times. In addition, the incorporation of two incubation cells in one chamber permits the study of two slices at nearly the same time.

The use of lung slices for measurement of bronchoconstriction was pioneered by DANDURAND et al. [7]. Based on their method, we have made the following improvements: 1) use of precision-cut lung slices; 2) incubation of the slices in a roller incubator; 3) development of a novel incubation chamber; and 4) omission of insulin and steroids from the medium. In general, compared to the study by DANDURAND et al. [7], these alterations clearly improved the precision of the measurements. For methacholine-induced bronchoconstriction, DANDURAND et al. [7] reported that $80 \%$ of the EC50 values were between $10^{-6}$ and $10^{-4} \mathrm{M}$, but the EC50 values that they obtained varied over five orders of magnitude. In the present study, the EC50 values varied only by two orders of magnitude at a given airway size (fig. $6 a)$, and the values obtained were highly reproducible (tables 1 and 2). The increased precision produced by the present method is probably due to the use of thinner slices and the omission of steroids. The technique made it possible to prepare lung slices that were much thinner than those described by DANDURAND et al. [7], i.e. $250 \pm 20 \mu \mathrm{m}$ compared to 500-1000 $\mu \mathrm{m}$. One advantage of thinner slices is that they permit study of smaller airways. Thinner slices produce less artefacts due to airways that are not cut exactly horizontally. The thinner the slices the less artefacts as a result of curved, branched airways or airways cut at an angle are obtained. In addition, the present data indicate that steroids increase the variability of the measurements (fig. 7).

One consequence of the improved precision was that we were able to show differences between small and medium-sized or large airways in their sensitivity towards methacholine. The data show a size-dependent constriction of airways. It was found that smaller airways were more sensitive to methacholine, a finding which is supported by previous in vivo [18-20] and in vitro $[21,22]$ studies. The present study differs from most previous studies in that the diameter of the airways that were examined was quite small, i.e. $0.035-0.58 \mathrm{~mm}$. To our knowledge, airways of this small size have only been studied by DANDURAND et al. [7], with, however, the limitations discussed above. Interestingly, we also found the same effect with thromboxane, i.e. that smaller airways are more sensitive to a constrictor agent [23]. The reason for the increased sensitivity of smaller airways is not clear, but the following suggestions have been made: 1) larger airways may release more quantities of an unknown bronchodilator [24]; 2) epithelium of smaller airways may be more permeable to drugs [21, $25,26]$; and 3 ) the orientation of smooth muscle in 
smaller airways may differ from that in larger ones [27]. However, since drugs may also reach airway smooth muscle directly in horizontally cut lung slices of only $250 \mu \mathrm{m}$, we do not believe that epithelial permeability is important in this model.

Thinner lung slices are also preferable because, as a consequence of the larger surface to tissue ratio, the supply of nutrients and oxygen is improved. The release of LDH and the incorporation of thymidine suggest that the slices were viable for at least $70 \mathrm{~h}$. With respect to function, the data for methacholine-induced bronchoconstriction showed similar responses for at least $28 \mathrm{~h}$ of incubation. The present investigation also showed that a dynamic organ culture system, i.e. a roller incubator, is superior to a static culture system. i.e. incubation in wells, as indicated by measurement of $\mathrm{LDH}$ leakage. This is supported by the fact that beating of cilia was observed for $72 \mathrm{~h}$ in the dynamic system, but only for $24 \mathrm{~h}$ in the static system. In addition, the staining of lung slices with trypan blue showed more destroyed cells in the static than in the dynamic system (data not shown). The use of the roller incubator provides constant movement of the medium and, hence, washing of the slices. It has been shown that continued submerging of slices in the medium may accelerate lung deterioration [19, 28]. Moreover, in vivo epithelial lung cells are separated from the ambient air only by a thin mucous film, and therefore a roller incubator, which regularly exposes the cells to air, may provide a more natural environment for these cells.

Since serum always introduces unknown variables, we avoided its addition to the medium. In long-term tissue and cell culture, serum-derived growth factors may be necessary to maintain proliferation. In the present system, however, this may not be required, since the incorporation of thymidine suggests continued proliferation in the culture. In addition, incubation with MTT showed that mitochondria were active in the lung slices for up to 3 days. MEM for cultivation of the slices, as used in the present study, has been shown to retain the histological structure of the tissue and to support deoxyribonucleic acid (DNA) and protein synthesis [3]. In contrast to DANDURAND et al. [7], the addition of insulin, hydrocortisone and vitamin A to the medium was omitted. We have not systematically explored the influence of these supplements to the viability of lung slices, but the prolonged viability of the lung slices, as discussed above, suggests that none of these supplements is essential to maintain survival of the lung slices.

With respect to airway contraction, we have investigated the effect of hydrocortisone on methacholine-induced bronchoconstriction. In the presence of the steroid, the EC50 value was increased, suggesting that steroidtreated airways are less sensitive to methacholine. This effect may be explained by the known inhibition of the catecholamine uptake system by steroids [29]. This would result in elevated tissue concentrations of noradrenaline, which in turn would act on $\beta_{2}$-receptors to relax airway smooth muscle. In addition, steroids increase the number of $\beta_{2}$-receptors on airway smooth cells $[30,31]$. To investigate the possibility that an enhanced $\beta_{2}$-receptor response causes the $\mathrm{HC}$-induced desensitization, steroid-treated slices were incubated with the $\beta$ receptor antagonist, propanolol. In these experiments, it was found that the effect of HC was largely attenuated by propanolol. Therefore, we conclude that the major part of the decreased responsiveness of steroid-treated airways may be explained by reduced uptake of noradrenaline and/or higher expression of $\beta_{2}$-receptors.

In summary, precision cut lung slices were cultured under serum- and steroid-free conditions in a roller incubator. These slices were then placed in a newly developed incubation chamber. The precise and reproducible measurement of airway contraction in individual airways made it possible to observe that smaller airways are more sensitive to methacholine.

Acknowledgements: The authors wish to thank G. Junge for excellent technical assistance, W. Schulter for the technical advice concerning the development of the incubation chamber, and M. Eltze for helpful discussions.

\section{References}

1. Krumdieck CL, dos Santos JE. Ho KJ. A new instrument for the rapid preparation of tissue slices. Anal Biochem 1980; 104: 118-123.

2. Brendel K, Gandolfi AJ, Krumdieck CL. Smith PF. Tissue slicing and culturing revisited. Trends Pharmacol Sci 1987; 8: 11-15.

3. Placke ME, Fisher GE. Adult peripheral lung organ culture: a model for respiratory tract toxicology. Pharmacology 1987; 90: 284-298.

4. Price RJ, Renwick AB, Beamand JA, et al. Comparison of the metabolism of 7-ethoxycumarin and cumarin in precision-cut rat liver and lung slices. Fund Chem Toxic 1995; 33: 233-237.

5. Fisher RL, Smith MS, Hasal SJ, Hasal KS, Gandolfi $\mathrm{AJ}$, Brendel $\mathrm{K}$. The use of human lung slices in toxicology. Hum Exp Toxicol 1994; 13: 466-471.

6. Stefaniak MS, Krumdieck CL, Spall RD, Gandolfi A, Brendel K. Biochemical and histological characterization of agar-filled precision-cut rat lung slices in dynamic organ culture as an in vitro tool. In Vitro Toxicol 1992; 5: 7-19.

7. Dandurand RJ, Wang CG, Phillips NC, Eidelman DH. Responsiveness of individual airway to methacholine in adult lung explants. J Appl Physiol 1993; 75: 364-372.

8. Drazen JM, Schneider MW. Comparative responses of tracheal spirals and parenchymal strips to histamine and carbachol in vitro. J Clin Invest 1978; 61: 1441-1447.

9. Evans JN. Adler KB. The lung strip: evaluation of a method to study contractility of pulmonary parenchyma. Exp Lung Res 1981; 2: 187-195.

10. Lulich KM, Mitchell HW, Sparrow MP. The cat lung strip as an in vitro preparation of peripheral airways: a comparison of $\beta$-adrenoceptor agonsists. autocoids and anaphylactic challenge on the lung strip and trachea. $\mathrm{Br}$ J Pharmacol 1976; 58: 71-79.

11. Uhlig S, von Bethmann NA, Featherstone RL, Wendel A. Pharmacologic characterization of endothelin receptor responses in the isolated perfused rat lung. $\mathrm{Am} \mathrm{J}$ Respir Crit Care Med 1995; 152: 1449-1460.

12. Uhlig S, Wollin L. An improved set-up for the isolated perfused rat lung. J Pharmacol Toxicol Meth 1994; 31: 85-94.

13. Horder M, Rej R. In: Bergmeyer HU, ed. Methods of Enzymatic Analysis. Weinheim, Germany, Verlag Chemie, 1984; pp. 444-449.

14. Mosman T. Rapid colorimetric assay for cell growth and 
survival: application to proliferation and cytotoxicity assay. J Immunol Meth 1983; 65: 55-56.

15. Lean D, Munson AP, Rodbard D. Simultaneous analysis of families of sigmoidal curves: application to bioassay. radioligand assay, and physical concentration-response curves. Am J Physiol 1978; 235: E97-E102.

16. Coles CJ, Edmondson DE, Singer TP. Inactivation of succinate dehydrogenase by 3-nitropropionate. J Biol Chem 1979; 254: 5161-5167.

17. Mansour S, Daniel EE. Maintenance of tone, role of arachidonate metabolites and effect of sensitization in guinea-pig trachea. Can J Physiol Pharmacol 1986; 64: 1096-1103.

18. Leff AR Munoz NM, Hendrix SG. Parasympathetic and adrenergic contractile responses in canine trachea and bronchus. J Appl Physiol 1983; 55: 113-120.

19. Bhatnager RS, Hussain MZ, Belton JC. Applications of lung organ culture in environmental investigations. In: Assessing Toxicity of Environmental Pollutants. Ann Arbor, MI, Ann Arbor Pub, pp. 121-149.

20. Shioya T, Solway J, Munoz NM, Mack M, Leff AR. Distribution of airway contractile responses within the major diameter bronchi during exogenous bronchoconstriction. Am Rev Respir Dis 1987; 135: 11051111.

21. Mitchell HW, Sparrow MP. Increased responsiveness to cholinergic stimulation of small compared to large diameter cartilaginous bronchi. Eur Respir J 1994; 7 : 298-305.

22. Gray PR, Mitchel HW. Effect of diameter on force generation and responsiveness of bronchial segments and rings. Eur Respir J 1996: 9: 500-505.

23. Martin C, Brasch F, Müller KM, Ullrich V, Uhlig S.
Thromboxane preferentially contracts smaller airways in rat lung. Am J Respir Crit Care Med 1996; 154: A687.

24. Stuart-Smith K, Vanhoutte PM. Heterogeneity in the effects of epithelium removal in the canine bronchial tree. J Appl Physiol 1987; 63: 2510-2515.

25. Borrowcliffe MP, Jones JG, Agnew JG, Francis RA, Clarke SW. The relative permeability of human conducting and terminal airways to $99 \mathrm{mTC}-\mathrm{DTPA}$. Eur $J$ Respir Dis 1987; 71: 68-77.

26. Hulsmann AR, Raatgeep HR, Den Hollander JC, Bakker WH, Saxena PR, De Jongste JC. Permeability of human isolated airways increases after hydrogen peroxide and poly-L-arginine. Am J Respir Crit Care Med 1996; 153: 841-846.

27. Ebina M, Yaegashi H, Takahashi T, Motomiya M, Tanemura M. Distribution of smooth muscles along the bronchial tree: a morphometric study of ordinary autopsy lungs. Am Rev Respir Dis 1990; 141: 1322-1326.

28. Williams PP, Gallagher JE. Preparation and long-term cultivation of porcine tracheal and lung organ cultures by alternate exposure to gaseous and liquid medium phases. In Vitro 1978; 14: 686-696.

29. Kalsner K. Mechanisms of inactivation of noradrenaline in the iris sphinter, tracheal muscle and facial artery of cattle: implications for adrenoceptor-mediated responses. Br J Pharmacol 1978; 64: 545-552.

30. Collins S, Caron MG, Lefkowitz RJ. $\beta_{2}$-adrenergic receptors in hamster smooth muscle cells are transcriptionally regulated by glucocorticoids. J Biol Chem 1988; 263: 9067-9070.

31. Mak JCW, Nishikawa M, Barnes P. Glucocorticosteroids increase $\beta_{2}$-adrenergic receptor transcription in human lung. Am J Physiol 1995; 268: L41-L46. 